\title{
Existence of Conformal Metrics with Prescribed Q-Curvature
}

\author{
Mohammed Ali Al-Ghamdi, ${ }^{1}$ Hichem Chtioui, ${ }^{1}$ and Afef Rigane ${ }^{2}$ \\ ${ }^{1}$ Department of Mathematics, King Abdulaziz University, P.O. Box 80230, Jeddah, Saudi Arabia \\ ${ }^{2}$ Department of Mathematics, Faculty of Sciences of Sfax, Road Soukra, Sfax, Tunisia
}

Correspondence should be addressed to Hichem Chtioui; hichem.chtioui@fss.rnu.tn

Received 13 October 2012; Revised 5 February 2013; Accepted 8 February 2013

Academic Editor: Svatoslav Staněk

Copyright (C) 2013 Mohammed Ali Al-Ghamdi et al. This is an open access article distributed under the Creative Commons Attribution License, which permits unrestricted use, distribution, and reproduction in any medium, provided the original work is properly cited.

We consider the problem of existence of conformal metrics with prescribed Q-curvature on standard sphere $S^{n}, n \geq 5$. Under the assumption that the order of flatness at critical points of prescribed Q-curvature function $K(x)$ is $\beta \in] 1, n-4]$, we give precise estimates on the losses of the compactness, and we prove new existence and multiplicity results through an Euler-Hopf type formula.

\section{Introduction and Main Result}

The Paneitz operator on $n$-Riemannian manifold $\left(M, g_{0}\right)$ is a fourth-order differential operator which arises in conformal geometry and satisfies a certain covariance property (see [1]). For $n \geq 5$ it is defined by

$$
P_{g_{0}}^{n} u=\Delta_{g_{0}}^{2} u-\operatorname{div}_{g_{0}}\left(a_{n} S_{g_{0}} g_{0}+b_{n} \operatorname{Ric}_{g_{0}}\right) d u+\frac{n-4}{2} Q_{g_{0}}^{n} u
$$

where

$$
\begin{gathered}
a_{n}=\frac{(n-2)^{2}+4}{2(n-1)(n-2)}, \quad b_{n}=-\frac{4}{n-2}, \\
Q_{g_{0}}^{n}=-\frac{1}{2(n-1)} \Delta_{g_{0}} S_{g_{0}}+\frac{n^{3}-4 n^{2}+16 n-16}{8(n-1)^{2}(n-2)^{2}} S_{g_{0}}^{2} \\
-\frac{2}{(n-2)^{2}}\left|\operatorname{Ric}_{g_{0}}\right|^{2} .
\end{gathered}
$$

Such a $Q_{g_{0}}^{n}$ is a fourth-order invariant called Q-curvature or Paneitz curvature. See $[2,3]$ for details about the properties of $P_{g_{0}}^{n}$.
If $g=u^{4 /(n-4)} g_{0}$ is a conformal metric to $g_{0}$, where $u$ is a smooth positive function, then the conformal covariance property of the Paneitz operator reads as follows:

$$
\forall \psi \in C^{\infty}(M): P_{g_{0}}^{n}(u \psi)=u^{(n+4) /(n-4)} P_{g}^{n}(\psi)
$$

If one prescribes the Q-curvature for the metric $g$ by a function $K$, this leads to the following equation:

$$
P_{g_{0}}^{n}(u)=u^{(n+4) /(n-4)} P_{g}^{n}(1)=\frac{n-4}{2} K u^{(n+4) /(n-4)} .
$$

The literature for the existence of solutions of the prescribed Q-curvature problem on compact manifolds is considerably bigger. In [4-7], existence results for the constant Qcurvature problem in 4-dimensional manifolds are given. On manifolds of dimension greater than 4 , existence results were given for Einstein manifolds in [3]. On the sphere $S^{n}$, we refer to results of [8-14] and the references therein.

In this paper we continue to study the problem of prescribing Q-curvature on the standard sphere $\left(S^{n}, g_{0}\right), n \geq$ 5 . The Paneitz operator in this case is coercive on the Sobolev space $H_{2}^{2}\left(S^{n}\right)$ and has the following expression:

$$
\mathscr{P}(u):=P_{g_{0}}^{n}(u)=\Delta_{g_{0}}^{2} u-c_{n} \Delta_{g_{0}} u+d_{n} u,
$$


where

$$
c_{n}=\frac{1}{2}\left(n^{2}-2 n-4\right), \quad d_{n}=\frac{n-4}{16} n\left(n^{2}-4\right) .
$$

According to problem (4), the problem can be formulated as follows. Given a smooth function $K$ on $S^{n}$, we look for solutions of

$$
\begin{gathered}
\mathscr{P}(u)=\frac{n-4}{2} K u^{(n+4) /(n-4)}, \\
u>0 \quad \text { on } S^{n} .
\end{gathered}
$$

The special case where the manifold is a sphere endowed with its standard metric deserves particular attention. Indeed due to Kazdan-Warner type obstructions, see [3], conditions have to be imposed on the function $K$.

To state our result we set up the following conditions and notation.

$(f)_{\beta}$ Assume that $K: S^{n} \rightarrow \mathbb{R}, n \geq 5$ be a $C^{1}$ positive function such that for any critical point $y$ of $K$, there exists some real number $\beta=\beta(y)$ such that in some geodesic normal coordinate system centered at $y$ (through the exponential map), we have

$$
K(x)=K(y)+\sum_{k=1}^{n} b_{k}\left|(x-y)_{k}\right|^{\beta}+R(x-y),
$$

where $b_{k}=b_{k}(y) \neq 0$, for all $k=1, \ldots, n, \sum_{k=1}^{n} b_{k} \neq 0$ and $\sum_{s=0}^{[\beta]}\left|\nabla^{s} R(x-y) \| x-y\right|^{-\beta+s}=o(1)$ as $x$ tends to $y$. Here $\nabla^{s}$ denotes all possible derivatives of order $s$ and $[\beta]$ is the integer part of $\beta$. For each critical point $y$ of $K$, we denote

$$
\widetilde{i}(y)=: \sharp\left\{b_{k}, k=1, \ldots, n \text {, such that } b_{k}<0\right\} \text {. }
$$

Let,

$$
\begin{gathered}
\mathscr{K}=\left\{y \in S^{n}, \nabla_{g_{0}} K(y)=0\right\} \\
\mathscr{K}^{+}=\left\{y \in \mathscr{K},-\sum_{k=1}^{n} b_{k}>0\right\}, \\
\mathscr{K}_{n-4}=\{y \in \mathscr{K}, \beta=\beta(y)=n-4\} .
\end{gathered}
$$

For each $p$-tuple, $p \geq 1$ of distinct points $\tau_{p}:=\left(y_{l_{1}}, \ldots, y_{l_{p}}\right)$ such that for all $i=1, \ldots, p, y_{l_{i}} \in \mathscr{K}^{+} \cap \mathscr{K}_{n-4}$, we define a $p \times p$ symmetric matrix $M\left(\tau_{p}\right)=\left(m_{i j}\right)$ by

$$
\begin{gathered}
m_{i i}=\frac{n-4}{n} \widetilde{c}_{1} \frac{-\sum_{k=1}^{n} b_{k}\left(y_{l_{i}}\right)}{K\left(y_{l_{i}}\right)^{n / 4}}, \\
m_{i j}=2^{(n-4) / 2} c_{1} \frac{-G\left(y_{l_{i}}, y_{l_{j}}\right)}{\left[K\left(y_{l_{i}}\right) K\left(y_{l_{j}}\right)\right]^{(n-4) / 8}},
\end{gathered}
$$

where

$$
\begin{gathered}
c_{1}=\int_{\mathbb{R}^{n}} \frac{d x}{\left(1+|x|^{2}\right)^{(n+4) / 2}}, \\
\widetilde{c}_{1}=c_{0}^{2 n /(n-4)} \int_{\mathbb{R}^{n}} \frac{\left|x_{1}\right|^{n-4}}{\left(1+|x|^{2}\right)^{n}} d x,
\end{gathered}
$$

and $G$ is the Green function for the operator $\mathscr{P}(u)$ on $S^{n}$. Here $x_{1}$ is the first component of $x$ in some geodesic normal coordinate system.

Let $\rho\left(\tau_{p}\right)$ be the least eigenvalue of $M\left(\tau_{p}\right)$. It was first pointed out by Bahri [15] that when the interaction between the different bubbles is of the same order as the self interaction, the least eigenvalue of some matrices like (11) plays a fundamental role in the existence of solutions to problems like (7). Regarding problem (7), such kind of phenomenon appears under $(f)_{\beta}$ condition in $\mathscr{K}_{n-4}$; see [11].

$\left(A_{1}\right)$ Assume that $\rho\left(\tau_{p}\right) \neq 0$ for each distinct point $y_{l_{1}}, \ldots, y_{l_{p}} \in \mathscr{K}^{+} \cap \mathscr{K}_{n-4}$.

Now, we introduce the following set:

$$
\begin{gathered}
\mathscr{C}_{\infty}:=\left\{\tau_{p}=\left(y_{l_{1}}, \ldots, y_{l_{p}}\right) \in\left(\mathscr{K}^{+}\right)^{p}, p \geq 1,\right. \\
\text { s.t. } y_{i} \neq y_{j} \forall i \neq j, \text { and if } \\
\left\{y_{l_{1}}, \ldots, y_{l_{p}}\right\} \cap \mathscr{K}_{n-4} \neq \emptyset,
\end{gathered}
$$

we denote by $y_{i_{1}}, \ldots, y_{i_{q}}$ all elements of

$$
\left\{y_{l_{1}}, \ldots, y_{l_{p}}\right\} \text { with } \beta\left(y_{i_{j}}\right)=n-4
$$

for each $j=1, \ldots, q$ then

$$
\left.\rho\left(y_{i_{1}}, \ldots, y_{i_{q}}\right)>0\right\} \text {. }
$$

The main result of this paper is the following.

Theorem 1. Assume that $K$ satisfies $\left(A_{1}\right)$ and $(f)_{\beta}$, with

$$
1<\beta \leq n-4 \text {. }
$$

If

$$
d=\sum_{\left(y_{1}, \ldots, y_{p}\right) \in \mathscr{C}_{\infty}}(-1)^{p-1+\sum_{k=1}^{p} n-\tilde{i}\left(y_{k}\right)}-1 \neq 0 .
$$

Then (7) has at least one solution.

Moreover if $(n-4) / 2<\beta \leq n-4$, for generic $K$ one has

$$
\sharp S \geq|d|,
$$

where $S$ denotes the set of solutions to (7).

Our argument uses a careful analysis of the lack of compactness of the Euler Lagrange functional $J$ associated with problem (7). Namely, we study the noncompact orbits of the gradient flow of $J$ the so-called critical points at infinity following the terminology of Bahri [15]. These critical points at infinity can be treated as usual critical points once a Morse lemma at infinity is performed from which we can derive just as in the classical Morse theory the difference of topology induced by these noncompact orbits and compute their Morse index. Such a Morse lemma at infinity is obtained through the construction of suitable pseudogradient for which the Palais-Smale condition is satisfied along the decreasing flow lines, as long as these flow lines do 
not enter the neighborhood of a finite number $y_{1}, \ldots, y_{p}$ of critical points of $K$ such that $\left(y_{1}, \ldots, y_{p}\right) \in \mathscr{C}_{\infty}$.

Similar Morse lemma at infinity has been established for the problem (7) on the sphere $S^{n}, n \geq 5$, under the hypothesis that the order of flatness at critical points of $K$ is $\beta \in[n-4, n[$; see [11].

The rest of this paper is organized as follows. In Section 2, we set up the variational problem and we recall the expansion of the gradient of the associated Euler-Lagrange functional near infinity. In Section 3, we characterize the critical points at infinity of the associated variational problem. Section 4 is devoted to the proof of the main result Theorem 1, while we give in Section 5 a more general statement than Theorem 1.

\section{General Framework and Some Known Facts}

2.1. Variational Structure and Lack of Compactness. Our problem (7) enjoys a variational structure. Indeed, solutions to (7) correspond to positive critical points of the functional

$$
J(u)=\frac{\int_{S^{n}} \mathscr{P} u u d v_{g_{0}}}{\left(\int_{S^{n}} K|u|^{2 n /(n-4)} d v_{g_{0}}\right)^{(n-4) / 2 n}},
$$

defined on

$$
\Sigma=\left\{u \in H_{2}^{2}\left(S^{n}\right),\|u\|^{2}:=\int_{S^{n}} \mathscr{P} u u d v_{g_{0}}=1\right\} .
$$

However, it is delicate from a variational viewpoint because the functional $J$ does not satisfy the Palais-Smale condition. This means that there exist sequences along which $J$ is bounded, its gradient goes to zero and which not converge. The analysis of sequences failing Palais-Smale condition can be analyzed along the ideas introduced in [16, pages 325 and 334]. In order to describe such a characterization in our case, we need to introduce some notations. For $a \in S^{n}$ and $\lambda>0$, let

$$
\begin{aligned}
\delta_{(a, \lambda)}(x)= & c_{0} \frac{1}{2^{(n-4) / 2}} \\
& \times \frac{\lambda^{(n-4) / 2}}{\left(1+\left(\left(\lambda^{2}-1\right) / 2\right)(1-\cos (d(x, a)))\right)^{(n-4) / 2}},
\end{aligned}
$$

where $d$ is the geodesic distance on $\left(S^{n}, g\right)$ and $c_{0}$ is chosen so that $\delta_{(a, \lambda)}$ is the family of solutions of the problem

$$
\mathscr{P}(u)=u^{(n+4) /(n-4)}, \quad u>0, \text { on } S^{n}
$$

(see [17]).

We define now the set of potential critical points at infinity associated with the function $J$. For $\varepsilon>0$ and $p \in \mathbb{N}^{*}$, let us define

$$
\begin{aligned}
& V(p, \varepsilon) \\
& =\left\{\begin{array}{l}
u \in \Sigma / \exists a_{1}, \ldots, a_{p} \in S^{n}, \exists \lambda_{1}, \ldots, \lambda_{p}>\varepsilon^{-1}, \\
\exists \alpha_{1}, \ldots, \alpha_{p}>0 \text { with }\left\|u-\sum_{i=1}^{p} \alpha_{i} \delta_{\left(a_{i}, \lambda_{i}\right)}\right\|<\varepsilon, \\
\left|J(u)^{n /(n-4)} \alpha_{i}^{8 /(n-4)} K\left(a_{i}\right)-1\right|<\varepsilon \forall i, \varepsilon_{i j}<\varepsilon \forall i \neq j .
\end{array}\right.
\end{aligned}
$$

Here, $\varepsilon_{i j}=\left[\left(\lambda_{i} / \lambda_{j}\right)+\left(\lambda_{j} / \lambda_{i}\right)+\left(\lambda_{i} \lambda_{j} / 2\right)(1-\right.$ $\left.\left.\cos d\left(a_{i}, a_{j}\right)\right)\right]^{-(n-4) / 2}$.

For $w$ a solution to (7) we also define $V(p, \varepsilon, w)$ as

$$
V(p, \varepsilon, w)=\left\{\begin{array}{l}
u \in \Sigma / \exists \alpha_{0}>0 \text { s.t. } u-\alpha_{0} w \in V(p, \varepsilon), \\
\left|\alpha_{0}^{8 /(n-4)} J(u)^{n /(n-4)}-1\right|<\varepsilon .
\end{array}\right.
$$

If $u$ is a function in $V(p, \varepsilon, w)$, one can find an optimal representation, following the ideas introduced in Proposition 5.2 of [15] and [16, pages 348-350]. Namely, we have the following.

Proposition 2. For any $p \in \mathbb{N}^{*}$, there is $\varepsilon_{p}>0$ such that if $\varepsilon \leq \varepsilon_{p}$ and $u \in V(p, \varepsilon, w)$, then the following minimization problem

$$
\min _{\substack{\alpha_{i}>0, \lambda_{i}>0, a_{i} \in S^{n}, h \in T_{w}\left(W_{u}(w)\right)}}\left\|u-\sum_{i=1}^{p} \alpha_{i} \delta_{\left(a_{i}, \lambda_{i}\right)}-\alpha_{0}(w+h)\right\|
$$

has a unique solution ( $\alpha, \lambda, a, h)$, up to a permutation.

In particular, we can write $u$ as follows:

$$
u=\sum_{i=1}^{p} \alpha_{i} \delta_{\left(a_{i}, \lambda_{i}\right)}+\alpha_{0}(w+h)+v
$$

where $v$ belongs to $H_{2}^{2}\left(S^{n}\right) \cap T_{w}\left(W_{s}(w)\right)$ and it satisfies $\left(V_{0}\right)$, $T_{w}\left(W_{u}(w)\right)$ and $T_{w}\left(W_{s}(w)\right)$ are the tangent spaces at $w$ of the unstable and stable manifolds of $w$ for a decreasing pseudogradient of $J$, and $\left(V_{0}\right)$ is the following:

$$
\begin{gathered}
\langle v, \psi\rangle=0 \quad \text { for } \psi \in\left\{\delta_{i}, \frac{\partial \delta_{i}}{\partial \lambda_{i}}, \frac{\partial \delta_{i}}{\partial a_{i}}, i=1, \ldots, p\right\} \\
\langle v, w\rangle=0, \\
\langle v, h\rangle=0 \quad \forall h \in T_{w} W_{u}(w) .
\end{gathered}
$$

Here, $\delta_{i}=\delta_{\left(a_{i}, \lambda_{i}\right)}$ and $\langle\cdot, \cdot\rangle$ denotes the scalar product defined on $H_{2}^{2}\left(S^{n}\right)$ by

$$
\begin{aligned}
\langle u, v\rangle= & \int_{S^{n}} \Delta_{g_{0}} u \Delta_{g_{0}} v d v_{g_{0}}+c_{n} \int_{S^{n}} \nabla_{g_{0}} u \nabla_{g_{0}} v d v_{g_{0}} \\
& +d_{n} \int_{S^{n}} u v d v_{g_{0}} .
\end{aligned}
$$

Notice that Proposition 2 is also true if we take $w=0$ and, therefore, $h=0$ and $u$ in $V(p, \varepsilon)$.

We are ready now to state the characterization of the sequences failing the Palais-Smale condition. For technical reasons, we introduce the following subsets of $\Sigma$. Let $\Sigma^{+}=$ $\{u \in \Sigma, u \geq 0\}$ and let for $\eta$ positive very small,

$$
V_{\eta}\left(\Sigma^{+}\right)=\left\{u \in \Sigma, J(u)^{(2 n-4) /(n-4)} e^{2 J(u)}\left\|u^{-}\right\|_{L^{2 n /(n-4)}}^{8 /(n-4)}<\eta\right\},
$$

where $u^{-}=\max (0,-u)$. Using the idea introduced in $[16,18]$, see also [19], we have the following proposition. 
Proposition 3. Let $\left(u_{k}\right)$ be a sequence in $V_{\eta}\left(\Sigma^{+}\right)$such that $J\left(u_{k}\right)$ is bounded and $\partial J\left(u_{k}\right)$ goes to zero. Then there exists an integer $p \in \mathbb{N}^{*}$, a sequence $\left(\varepsilon_{K}\right)>0$, $\varepsilon_{k}$ tends to zero, and an extracted subsequence of $u_{k}$ 's, again denoted $\left(u_{k}\right)$, such that $u_{k} \in V\left(p, \varepsilon_{k}, w\right)$ where $w$ is zero or a solution to (7).

Now arguing as in [16] (pages 326, 327, and 334), we have the following Morse lemma which completely gets rid of the $v$-contributions and shows that it can be neglected with respect to the concentration phenomenon.

Proposition 4. There is a $\mathscr{C}^{1}$-map which to each $\left(\alpha_{i}, a_{i}, \lambda_{i}, h\right)$ such that $\sum_{i=1}^{p} \alpha_{i} \delta_{\left(a_{i}, \lambda_{i}\right)}+\alpha_{0}(w+h)$ belongs to $V(p, \varepsilon, w)$ associates $\bar{v}=\bar{v}(\alpha, a, \lambda, h)$ such that $\bar{v}$ is unique and satisfies

$$
\begin{aligned}
& J\left(\sum_{i=1}^{p} \alpha_{i} \delta_{\left(a_{i}, \lambda_{i}\right)}+\alpha_{0}(w+h)+\bar{v}\right) \\
& \quad=\min _{v \in\left(V_{0}\right)}\left\{J\left(\sum_{i=1}^{p} \alpha_{i} \delta_{\left(a_{i}, \lambda_{i}\right)}+\alpha_{0}(w+h)+v\right)\right\} .
\end{aligned}
$$

Moreover, there exists a change of variables $v-\bar{v} \rightarrow V$ such that

$$
\begin{aligned}
& J\left(\sum_{i=1}^{p} \alpha_{i} \delta_{\left(a_{i}, \lambda_{i}\right)}+\alpha_{0}(w+h)+v\right) \\
& \quad=J\left(\sum_{i=1}^{p} \alpha_{i} \delta_{\left(a_{i}, \lambda_{i}\right)}+\alpha_{0}(w+h)+\bar{v}\right)+\|V\|^{2} .
\end{aligned}
$$

The following proposition gives precise estimates of $\bar{v}$.

Proposition 5 (see [11, Lemma 3.1]). Let $u=\sum_{i=1}^{p} \alpha_{i} \delta_{i}+$ $\alpha_{0}(w+h) \in V(p, \varepsilon, w)$ and let $\bar{v}$ be defined in Proposition 4. One has the following estimates: there exists $c>0$ independent of u such that the following holds:

$$
\begin{aligned}
&\|\bar{v}\| \leq c \sum_{i=1}^{p}\left[\frac{1}{\lambda_{i}^{n / 2}}+\frac{1}{\lambda_{i}^{\beta}}+\frac{\left|\nabla K\left(a_{i}\right)\right|}{\lambda_{i}}+\frac{\left(\log \lambda_{i}\right)^{(n+4) / 2 n}}{\lambda_{i}^{(n+4) / 2}}\right] \\
&+c \begin{cases}\sum_{k \neq r} \varepsilon_{k r}^{(n+4) / 2(n-4)}\left(\log \varepsilon_{k r}^{-1}\right)^{(n+4) / 2 n}, & \text { if } n \geq 12 \\
\sum_{k \neq r} \varepsilon_{k r}\left(\log \varepsilon_{k r}^{-1}\right)^{(n-4) / n}, & \text { if } n<12 .\end{cases}
\end{aligned}
$$

At the end of this subsection, we give the following definition.

Definition 6 (see $[16,18])$. A critical point at infinity of $J$ on $V_{\eta}\left(\Sigma^{+}\right)$is a limit of a flow line $u(s)$ of the equation

$$
\begin{gathered}
\frac{\partial u}{\partial s}=-\partial J(u(s)), \\
u(0)=u_{0},
\end{gathered}
$$

such that $u(s)$ remains in $V(p, \varepsilon(s), w)$ for $s \geq s_{0}$. Here $w$ is either zero or a solution to (7) and $\varepsilon(s)$ is some positive function tending to zero when $s \rightarrow+\infty$. Using Proposition $2, u(s)$ can be written as

$$
u(s)=\sum_{i=1}^{p} \alpha_{i}(s) \delta_{\left(a_{i}(s), \lambda_{i}(s)\right)}+\alpha_{0}(s)(w+h(s))+v(s)
$$

Denoting $\widetilde{\alpha}_{i}:=\lim _{s \rightarrow+\infty} \alpha_{i}(s), \tilde{y}_{i}:=\lim _{s \rightarrow+\infty} a_{i}(s)$, we denote by

$$
\sum_{i=1}^{p} \widetilde{\alpha}_{i} \delta_{\left(\tilde{y}_{i}, \infty\right)}+\widetilde{\alpha}_{0} w \quad \text { or } \quad\left(\tilde{y}_{1}, \ldots, \tilde{y}_{p}, w\right)_{\infty}
$$

such a critical point at infinity. If $w \neq 0$, it is called of $w$-type or mixed type.

Notice that $V_{\eta}\left(\Sigma^{+}\right)$remains invariant under the flow generated by $(-\partial J)$ (see Lemma 5.1 of [20]; see also Lemma 4.1 of [18]).

2.2. Expansion of the Gradient of the Functional. In this subsection, we recall the expansion of the gradient of the functional $J$ in $V(p, \varepsilon), p \geq 1$.

Proposition 7 (see [11]). For any $u=\sum_{j=1}^{p} \alpha_{j} \delta_{j}$ in $V(p, \varepsilon)$, the following expansion holds

(i)

$$
\begin{aligned}
\left\langle\partial J(u), \lambda_{i} \frac{\partial \delta_{i}}{\partial \lambda_{i}}\right\rangle= & -2 c_{2} J(u) \sum_{i \neq j} \alpha_{j} \lambda_{i} \frac{\partial \varepsilon_{i j}}{\partial \lambda_{i}} \\
& +o\left(\sum_{i \neq j} \varepsilon_{i j}\right)+o\left(\frac{1}{\lambda_{i}}\right),
\end{aligned}
$$

where $c_{2}=c_{0}^{2 n /(n-4)} \int_{\mathbb{R}^{n}} d y /\left(1+|y|^{2}\right)^{(n+4) / 2}$.

(ii) If $a_{i} \in B\left(y_{j_{i}}, \rho\right), y_{j_{i}} \in \mathscr{K}$ and $\rho$ is a positive constant small enough, one has

$$
\begin{aligned}
\left\langle\partial J(u), \lambda_{i} \frac{\partial \delta_{i}}{\partial \lambda_{i}}\right\rangle & \\
=2 J(u)[ & -c_{2} \sum_{j \neq i} \alpha_{j} \lambda_{i} \frac{\partial \varepsilon_{i j}}{\partial \lambda_{i}}+\frac{n-4}{2 n} c_{0}^{2 n /(n-4)} \beta \frac{\alpha_{i}}{K\left(a_{i}\right)} \\
& \times \frac{\sum_{k=1}^{n} b_{k}}{\lambda_{i}^{\beta}} \int_{\mathbb{R}^{n}} \operatorname{signe}\left(x_{k}+\lambda_{i}\left(a_{i}-y_{j_{i}}\right)_{k}\right) \\
& \times\left|x_{k}+\lambda_{i}\left(a_{i}-y_{j_{i}}\right)_{k}\right|^{\beta-1} \\
& \times \frac{x_{k}}{\left(1+|x|^{2}\right)^{n}} d x \\
& \left.+o\left(\sum_{j \neq i} \varepsilon_{i j}+\sum_{j=1}^{p} \frac{1}{\lambda_{j}^{\beta}}\right)\right] .
\end{aligned}
$$


(iii) Furthermore if $\lambda_{i}\left|a_{i}-y_{j_{i}}\right|<\delta$, for $\delta$ very small, one then has

$$
\begin{aligned}
\left\langle\partial J(u), \lambda_{i} \frac{\partial \delta_{i}}{\partial \lambda_{i}}\right\rangle & \\
=2 J(u) \times & {\left[\frac{n-4}{2 n} \beta c_{3} \frac{\alpha_{i}}{K\left(a_{i}\right)} \frac{\sum_{k=1}^{n} b_{k}}{\lambda_{i}^{\beta}}-c_{2} \sum_{j \neq i} \alpha_{j} \lambda_{i} \frac{\partial \varepsilon_{i j}}{\partial \lambda_{i}}\right.} \\
& \left.+o\left(\sum_{j \neq i} \varepsilon_{i j}+\sum_{j=1}^{p} \frac{1}{\lambda_{j}^{\beta}}\right)\right]
\end{aligned}
$$

where $c_{3}=c_{0}^{2 n /(n-4)} \int_{S^{n}}\left|x_{1}\right|^{\beta} /\left(1+|x|^{2}\right)^{n} d x$.

Proposition 8 (see [11]). Letting $u=\sum_{j=1}^{p} \alpha_{j} \delta_{j} \in V(p, \varepsilon)$, one has

(i)

$$
\begin{aligned}
\langle\partial J(u), & \left.\frac{1}{\lambda_{i}} \frac{\partial \delta_{i}}{\partial a_{i}}\right\rangle \\
= & -c_{5}(J(u))^{2(n-2) /(n-4)} \alpha_{i}^{(n+4) /(n-4)} \frac{\nabla K\left(a_{i}\right)}{\lambda_{i}} \\
& +O\left(\sum_{i \neq j} \frac{1}{\lambda_{i}}\left|\frac{\partial \varepsilon_{i j}}{\partial a_{i}}\right|\right)+o\left(\sum_{i \neq j} \varepsilon_{i j}+\frac{1}{\lambda_{i}}\right),
\end{aligned}
$$

where $c_{5}=\int_{\mathbb{R}^{n}} d y /\left(1+|y|^{2}\right)^{n}$.

(ii) If $a_{i} \in B\left(y_{j_{i}}, \rho\right), y_{j_{i}} \in \mathscr{K}$, one has

$$
\begin{aligned}
\langle\partial J(u) & \left., \frac{1}{\lambda_{i}} \frac{\partial \delta_{i}}{\partial\left(a_{i}\right)_{k}}\right\rangle \\
= & -2(n-4) c_{0}^{2 n /(n-4)} \alpha_{i}^{(n+4) /(n-4)}(J(u))^{2(n-2) /(n-4)} \frac{1}{\lambda_{i}^{\beta}} \\
& \times \int_{\mathbb{R}^{n}} b_{k}\left|x_{k}+\lambda_{i}\left(a_{i}-y_{j_{i}}\right)_{k}\right|^{\beta} \frac{x_{k}}{\left(1+|x|^{2}\right)^{n+1}} d y \\
& +o\left(\sum_{i \neq j} \varepsilon_{i j}\right)+o\left(\sum_{i=1}^{p} \frac{1}{\lambda_{i}^{\beta}}\right)+O\left(\sum_{i \neq j} \frac{1}{\lambda_{i}}\left|\frac{\partial \varepsilon_{i j}}{\partial a_{i}}\right|\right),
\end{aligned}
$$

where $k=1, \ldots, n$ and $\left(a_{i}\right)_{k}$ is the $k$ th component of $a_{i}$ in some geodesic normal coordinates system.

\section{Characterization of the Critical Points at Infinity}

This section is devoted to the characterization of the critical points at infinity in $V(p, \varepsilon), p \geq 1$, under $\beta$-flatness condition with $1<\beta \leq n-4$. This characterization is obtained through the construction of a suitable pseudogradient at infinity for which the Palais-Smale condition is satisfied along the decreasing flow-lines as long as these flow lines do not enter in the neighborhood of finite number of critical points $y_{i}, i=1, \ldots, p$ of $K$ such that $\left(y_{1}, \ldots, y_{p}\right) \in \mathscr{C}_{\infty}$.

Now we introduce the following main result.

Theorem 9. Assume that $K$ satisfies $\left(A_{1}\right)$ and $(f)_{\beta}, 1<\beta \leq$ $n-4$.

Let $\beta:=\max \{\beta(y) / y \in \mathscr{K}\}$. For $p \geq 1$, there exists $a$ pseudogradient $W$ in $V(p, \varepsilon)$ so that the following holds.

There exists a constant $c>0$ independent of $u=$ $\sum_{i=1}^{p} \alpha_{i} \delta_{\left(a_{i} \lambda_{i}\right)} \in V(p, \varepsilon)$ such that

$$
\begin{aligned}
& \langle\partial J(u), W(u)\rangle \\
& \leq-c\left(\sum_{i=1}^{p} \frac{1}{\lambda_{i}^{\beta}}+\sum_{i=1}^{p} \frac{\left|\nabla K\left(a_{i}\right)\right|}{\lambda_{i}}+\sum_{j \neq i} \varepsilon_{i j}\right) . \\
& \left\langle\partial J(u+\bar{v}), W(u)+\frac{\partial \bar{v}}{\partial\left(\alpha_{i}, a_{i}, \lambda_{i}\right)}(W(u))\right\rangle \\
& \leq-c\left(\sum_{i=1}^{p} \frac{1}{\lambda_{i}^{\beta}}+\sum_{i=1}^{p} \frac{\left|\nabla K\left(a_{i}\right)\right|}{\lambda_{i}}+\sum_{j \neq i} \varepsilon_{i j}\right) .
\end{aligned}
$$

Furthermore $|W|$ is bounded and the only case where the maximum of the $\lambda_{i}$ 's is not bounded is when $a_{i} \in B\left(y_{l_{i}}, \rho\right)$ with $y_{l_{i}} \in \mathscr{K}$, for all $i=1, \ldots, p,\left(y_{l_{1}}, \ldots, y_{l_{p}}\right) \in \mathscr{C}_{\infty}$.

We will prove Theorem 9 at the end of the section. Now we state two results which deal with two specific cases of Theorem 9. Let

$$
\begin{gathered}
V_{1}(p, \varepsilon)=\left\{u=\sum_{i=1}^{p} \alpha_{i} \delta_{i} \in V(p, \varepsilon) \text { s.t., } a_{i} \in B\left(y_{l_{i}}, \rho\right),\right. \\
\left.y_{l_{i}} \in \mathscr{K} \backslash \mathscr{K}_{n-4} \forall i=1, \ldots, p\right\}, \\
V_{2}(p, \varepsilon)=\left\{\begin{array}{c}
u=\sum_{i=1}^{p} \alpha_{i} \delta_{i} \in V(p, \varepsilon) \text { s.t., } a_{i} \in B\left(y_{l_{i}}, \rho\right), \\
\left.y_{l_{i}} \in \mathscr{K}_{n-4} \forall i=1, \ldots, p\right\} .
\end{array}\right.
\end{gathered}
$$

We then have the following.

Proposition 10 (see [11], Proposition 3.7). For $p \geq 1$ there exists a pseudogradient $W_{2}$ in $V_{2}(p, \varepsilon)$ such that for all $u=$ $\sum_{i=1}^{p} \alpha_{i} \delta_{i} \in V_{2}(p, \varepsilon)$, one has

$$
\left\langle\partial J(u), W_{2}(u)\right\rangle \leq-c\left(\sum_{i=1}^{p} \frac{1}{\lambda_{i}^{n-4}}+\sum_{i \neq j} \varepsilon_{i j}+\sum_{i=1}^{p} \frac{\left|\nabla K\left(a_{i}\right)\right|}{\lambda_{i}}\right),
$$

where $c$ is a positive constant independent of $u$. Furthermore, one has $\left|W_{2}\right|$ which is bounded and the only case where the maximum of $\lambda_{i}$ 's is not bounded is when $a_{i} \in B\left(y_{l_{i}}, \rho\right), y_{l_{i}} \in$ $\mathscr{K}^{+}$, for all $i=1, \ldots, p$, with $\rho\left(y_{l_{1}}, \ldots, y_{l_{p}}\right)>0$. 
Proposition 11. For $p \geq 1$, there exists a pseudogradient $W_{1}$ in $V_{1}(p, \varepsilon)$ so that the following holds.

There exists $c>0$ independent of $u=\sum_{i=1}^{p} \alpha_{i} \delta_{i} \in V_{1}(p, \varepsilon)$ such that

$$
\left\langle\partial J(u), W_{1}(u)\right\rangle \leq-c\left(\sum_{i=1}^{p} \frac{1}{\lambda_{i}^{\beta}}+\sum_{i \neq j} \varepsilon_{i j}+\sum_{i=1}^{p} \frac{\left|\nabla K\left(a_{i}\right)\right|}{\lambda_{i}}\right) .
$$

Furthermore $\left|W_{1}\right|$ is bounded and the only case where the maximum of the $\lambda_{i}$ 's is not bounded is when $a_{i} \in B\left(y_{l_{i}}, \rho\right)$ with $y_{l_{i}} \in \mathscr{K}^{+}$, for all $i=1, \ldots, p$, and $y_{i} \neq y_{j}$ for all $i \neq j$.

Observe that in $V_{1}(p, \varepsilon)$ the interaction of two bubbles is negligible with respect to the self-interaction. Similar phenomena occur for the scalar curvature problem, see [21], so the proof of Proposition 11 is similar to the corresponding statement in [21].

Before giving the proof of Theorem 9, we state the following notations extracted from [11].

Let $M_{1}$ be a fixed positive constant large enough and let $u=\sum_{i=1}^{p} \alpha_{i} \delta_{\left(a_{i}, \lambda_{i}\right)} \in V(p, \varepsilon)$ such that $a_{i} \in B\left(a_{i}, \rho\right), y_{l_{i}} \in \mathscr{K}$, for all $i=1, \ldots, p$. For any index $i, 1 \leq i \leq p$, we define the following vector fields:

$$
\begin{gathered}
Z_{i}(u)=\alpha_{i} \lambda_{i} \frac{\partial \delta_{\left(a_{i}, \lambda_{i}\right)}}{\partial \lambda_{i}} \\
X_{i}=\alpha_{i} \sum_{k=1}^{n} \frac{1}{\lambda_{i}} \frac{\partial \delta_{\left(a_{i}, \lambda_{i}\right)}}{\partial\left(a_{i}\right)_{k}} \int_{\mathbb{R}^{n}} b_{k} \frac{\left|x_{k}+\lambda_{i}\left(a_{i}-y_{l_{i}}\right)_{k}\right|^{\beta_{i}}}{\left(1+\lambda_{i}\left|\left(a_{i}-y_{l_{i}}\right)_{k}\right|\right)^{\beta_{i}-1}} \\
\times \frac{x_{k}}{\left(1+|x|^{2}\right)^{n+1}} d x
\end{gathered}
$$

We claim that $X_{i}$ is bounded. Indeed, the claim is trivial if $\lambda_{i}\left|a_{i}-y_{l_{i}}\right| \leq M_{1}$. If $\lambda_{i}\left|a_{i}-y_{l_{i}}\right|>M_{1}$, for any $k, 1 \leq k \leq n$, such that $\lambda_{i}\left|\left(a_{i}-y_{l_{i}}\right)_{k}\right|>M_{1} / \sqrt{n}$, we have the following estimate:

$$
\begin{aligned}
\int_{\mathbb{R}^{n}} \frac{\left|x_{k}+\lambda_{i}\left(a_{i}-y_{l_{i}}\right)_{k}\right|^{\beta_{i}} x_{k}}{\left(1+|x|^{2}\right)^{n+1}} d x \\
=c\left(\text { signe } \lambda_{i}\left(a_{i}-y_{l_{i}}\right)_{k}\right)\left(\lambda_{i}\left|\left(a_{i}-y_{l_{i}}\right)_{k}\right|\right)^{\beta_{i}-1} \\
\quad \times(1+o(1)) .
\end{aligned}
$$

Hence our claim follows. Next, we will say that

$$
\begin{aligned}
& i \in L_{1} \quad \text { if } \lambda_{i}\left|a_{i}-y_{l_{i}}\right| \leq M_{1}, \\
& i \in L_{2} \quad \text { if } \lambda_{i}\left|a_{i}-y_{l_{i}}\right|>M_{1},
\end{aligned}
$$

and we will denote by $k_{i}$ the index satisfying

$$
\left|\left(a_{i}-y_{l_{i}}\right)_{k_{i}}\right|=\max _{1 \leq k \leq n}\left|\left(a_{i}-y_{l_{i}}\right)_{k}\right| \text {. }
$$

It easy to see that if $i \in L_{2}$, then $\lambda_{i}\left|\left(a_{i}-y_{l_{i}}\right)_{k_{i}}\right|>M_{1} / \sqrt{n}$.
Now, we introduce the following two lemmas.

Lemma 12. Let $u=\sum_{i=1}^{p} \alpha_{i} \delta_{\left(a_{i}, \lambda_{i}\right)} \in V(p, \varepsilon)$, such that $a_{i} \in$ $B\left(y_{l_{i}}, \rho\right), y_{l_{i}} \in \mathscr{K}$, for all $i=1, \ldots, p$. One then has

$$
\begin{aligned}
\left\langle\partial J(u), Z_{i}(u)\right\rangle= & -2 c_{2} J(u) \sum_{j \neq i} \alpha_{i} \alpha_{j} \frac{\partial \varepsilon_{i j}}{\partial \lambda_{i}}+O\left(\frac{1}{\lambda_{i}^{\beta_{i}}}\right) \\
& +\left[O\left(\frac{\left|\left(a_{i}-y_{l_{i}}\right)_{k_{i}}\right|^{\beta_{i}-2}}{\lambda_{i}^{2}}\right), \text { if } i \in L_{2}\right] \\
& +o\left(\sum_{j \neq i} \varepsilon_{i j}\right)+o\left(\sum_{j=1}^{p} \frac{1}{\lambda_{j}^{\beta_{j}}}\right),
\end{aligned}
$$

where $k_{i}$ is defined in (45).

Proof. Using Proposition 7, we have

$$
\begin{aligned}
& \left\langle\partial J(u), Z_{i}(u)\right\rangle \\
& =-2 c_{2} J(u) \sum_{j \neq i} \alpha_{i} \alpha_{j} \lambda_{i} \frac{\partial \varepsilon_{i j}}{\partial \lambda_{i}}+\frac{n-4}{2 n} c_{0}^{2 n /(n-4)} \beta \frac{\alpha_{i}^{2}}{K\left(a_{i}\right)} \\
& \quad \times \int_{\mathbb{R}^{n}} \operatorname{signe}\left(x_{k}+\lambda_{i}\left(a_{i}-y_{j_{i}}\right)_{k}\right)\left|x_{k}+\lambda_{i}\left(a_{i}-y_{j_{i}}\right)_{k}\right|^{\beta-1} \\
& \quad \times \frac{x_{k}}{\left(1+|x|^{2}\right)^{n}} d x+o\left(\sum_{j \neq i} \varepsilon_{i j}\right)+o\left(\sum_{j=1}^{p} \frac{1}{\lambda_{j}^{\beta_{j}}}\right) .
\end{aligned}
$$

Observe that for $k \in\{1, \ldots, n\}$, if $\lambda_{i}\left|\left(a_{i}-y_{l_{i}}\right)_{k}\right|>M_{1} / \sqrt{n}$, we have

$$
\begin{aligned}
& \int_{\mathbb{R}^{n}} \operatorname{signe}\left(x_{k}+\lambda_{i}\left(a_{i}-y_{j_{i}}\right)_{k}\right) \\
& \times \frac{\left|x_{k}+\lambda_{i}\left(a_{i}-y_{l_{i}}\right)_{k}\right|^{\beta_{i}-1} x_{k}}{\left(1+|x|^{2}\right)^{n}} d x \\
& =c \operatorname{signe}\left(\lambda_{i}\left(a_{i}-y_{j_{i}}\right)_{k}\right) \\
& \quad \times\left(\lambda_{i}\left|\left(a_{i}-y_{l_{i}}\right)_{k}\right|\right)^{\beta_{i}-2}(1+o(1)),
\end{aligned}
$$

taking $M_{1}$ large enough. If not, we have

$$
\int_{\mathbb{R}^{n}} \frac{\left|x_{k}+\lambda_{i}\left(a_{i}-y_{l_{i}}\right)_{k}\right|^{\beta_{i}-1}\left|x_{k}\right|}{\left(1+|x|^{2}\right)^{n}} d x=O(1) .
$$

Using the fact that $k_{i}$ defined in (45) satisfies $\lambda_{i}\left|\left(a_{i}-y_{l_{i}}\right)_{k_{i}}\right|>$ $M_{1} / \sqrt{n}$, if $i \in L_{2}$, Lemma 12 follows. 
Lemma 13. For $u=\sum_{i=1}^{p} \alpha_{i} \delta_{\left(a_{i}, \lambda_{i}\right)} \in V(p, \varepsilon)$, such that $a_{i} \in$ $B\left(y_{l_{i}}, \rho\right), y_{l_{i}} \in \mathscr{K}$, for all $i=1, \ldots, p$, one has

$$
\begin{aligned}
\left\langle\partial J(u), X_{i}(u)\right\rangle & \\
\leq & O\left(\sum_{j \neq i} \frac{1}{\lambda_{i}}\left|\frac{\partial \varepsilon_{i j}}{\partial a_{i}}\right|\right)+O\left[\left(\frac{1}{\lambda_{i}^{\beta_{i}}}\right), \text { if } i \in L_{1}\right] \\
& +\left[-c\left(\frac{1}{\lambda_{i}^{\beta_{i}}}+\frac{\left|\left(a_{i}-y_{l_{i}}\right)_{k_{i}}\right|}{\lambda_{i}}\right), \text { if } i \in L_{2}\right] \\
& +o\left(\sum_{j=1}^{p} \frac{1}{\lambda_{j}^{\beta_{j}}}\right)
\end{aligned}
$$

where $k_{i}$ is defined in (45).

Proof. Using Proposition 8, we have

$$
\begin{aligned}
& \left\langle\partial J(u), X_{i}(u)\right\rangle \\
& =-2(n-4) c_{0}^{2 n /(n-4)} \alpha_{i}^{2 n /(n-4)} \frac{1}{\lambda_{i}^{\beta_{i}}} \\
& \times \sum_{k=1}^{n}\left(\int_{\mathbb{R}^{n}} b_{k} \frac{\left|x_{k}+\lambda_{i}\left(a_{i}-y_{l_{i}}\right)_{k}\right|^{\beta_{i}}}{\left(1+\lambda_{i}\left|\left(a_{i}-y_{l_{i}}\right)_{k}\right|\right)^{\left(\beta_{i}-1\right) / 2}}\right. \\
& \left.\times \frac{x_{k}}{\left(1+|x|^{2}\right)^{n+1}} d x\right)^{2} \\
& +O\left(\sum_{j \neq i} \frac{1}{\lambda_{i}}\left|\frac{\partial \varepsilon_{i j}}{\partial a_{i}}\right|\right)+o\left(\sum_{j=1}^{p} \frac{1}{\lambda_{j}^{\beta_{j}}}\right) \\
& \leq-c \frac{1}{\lambda_{i}^{\beta_{i}}}\left(\int_{\mathbb{R}^{n}} b_{k_{i}} \frac{\left|x_{k}+\lambda_{i}\left(a_{i}-y_{l_{i}}\right)_{k_{i}}\right|^{\beta_{i}}}{\left(1+\lambda_{i}\left|\left(a_{i}-y_{l_{i}}\right)_{k_{i}}\right|\right)^{\left(\beta_{i}-1\right) / 2}}\right. \\
& \left.\times \frac{x_{k_{i}}}{\left(1+|x|^{2}\right)^{n+1}} d x\right)^{2} \\
& +O\left(\sum_{j \neq i} \frac{1}{\lambda_{i}}\left|\frac{\partial \varepsilon_{i j}}{\partial a_{i}}\right|\right)+o\left(\sum_{j=1}^{p} \frac{1}{\lambda_{j}^{\beta_{j}}}\right) .
\end{aligned}
$$

Using (43) and the fact that $\lambda_{i}\left|\left(a_{i}-y_{l_{i}}\right)_{k_{i}}\right|>M_{1} / \sqrt{n}$, if $i \in L_{2}$, Lemma 13 follows.

Proof of Theorem 9. In order to complete the construction of the pseudogradient $W$ suggested in Theorem 9, it only remains (using Propositions 10 and 11) to focus attention on the two following subsets of $V(p, \varepsilon)$.

Subset 1. We consider here the case of $u=\sum_{i=1}^{p} \alpha_{i} \delta_{\left(a_{i} \lambda_{i}\right)}=$ $\sum_{i \in I_{1}} \alpha_{i} \delta_{\left(a_{i} \lambda_{i}\right)}+\sum_{i \in I_{2}} \alpha_{i} \delta_{\left(a_{i} \lambda_{i}\right)}$ such that

$$
\begin{gathered}
I_{1} \neq \emptyset, \quad I_{2} \neq \emptyset, \quad \sum_{i \in I_{1}} \alpha_{i} \delta_{\left(a_{i} \lambda_{i}\right)} \in V_{1} \quad\left(\sharp I_{1}, \varepsilon\right), \\
\sum_{i \in I_{2}} \alpha_{i} \delta_{\left(a_{i} \lambda_{i}\right)} \in V_{2} \quad\left(\sharp I_{2}, \varepsilon\right) .
\end{gathered}
$$

Without loss of generality, we can assume that

$$
\lambda_{1} \leq \cdots \leq \lambda_{p}
$$

We distinguish three cases.

Case 1. $u_{1}:=\sum_{i \in I_{1}} \alpha_{i} \delta_{\left(a_{i} \lambda_{i}\right)} \notin V_{1}^{1}\left(\sharp I_{1}, \varepsilon\right)=\{u=$ $\sum_{j=1}^{\sharp I_{1}} \alpha_{j} \delta_{\left(a_{j}, \lambda_{j}\right)}, a_{j} \in B\left(y_{l_{j}}, \rho\right), y_{l_{j}} \in \mathscr{K}^{+}$for all $j=1, \ldots, \sharp I_{1}$ and $y_{l_{j}} \neq y_{l_{k}}$ for all $\left.j \neq k\right\}$.

Let $\widetilde{W_{1}}$ be the pseudogradient on $V(p, \varepsilon)$ defined by $\widetilde{W_{1}}(u)=W_{1}\left(u_{1}\right)$, where $W_{1}$ is the vector filed defined by Proposition 11 in $V_{1}\left(\sharp I_{1}, \varepsilon\right)$. Note that if $u_{1} \notin V_{1}^{1}\left(\sharp I_{1}, \varepsilon\right)$, then the pseudo-gradient $W_{1}\left(u_{1}\right)$ does not increase the maximum of the $\lambda_{i}$ 's, $i \in I_{1}$. Using Proposition 11, we have

$$
\begin{aligned}
\left\langle\partial J(u), \widetilde{W}_{1}(u)\right\rangle & \\
\leq & -c\left(\sum_{i \in I_{1}} \frac{1}{\lambda_{i}^{\beta_{i}}}+\sum_{j \neq i, i, j \in I_{1}} \varepsilon_{i j}+\sum_{i \in I_{1}} \frac{\left|\nabla K\left(a_{i}\right)\right|}{\lambda_{i}}\right) \\
& +O\left(\sum_{i \in I_{1}, j \in I_{2}} \varepsilon_{i j}\right) .
\end{aligned}
$$

An easy calculation yields

$$
\varepsilon_{i j}=O\left(\frac{1}{\left(\lambda_{i} \lambda_{j}\right)^{(n-4) / 2}}\right)=o\left(\frac{1}{\lambda_{i}^{\beta_{i}}}\right)+o\left(\frac{1}{\lambda_{j}^{\beta_{j}}}\right)
$$

Fix $i_{0} \in I_{1}$, we denote by

$$
J_{1}=\left\{i \in I_{2} \text {, s.t, } \lambda_{i}^{n-4} \geq \frac{1}{2} \lambda_{i_{0}}^{\beta_{i_{0}}}\right\}, \quad J_{2}=I_{2} \backslash J_{1} \text {. }
$$

Using (54) and (55), we find that

$$
\begin{aligned}
& \left\langle\partial J(u), \widetilde{W}_{1}(u)\right\rangle \\
& \leq-c\left(\sum_{i \in I_{1} \cup J_{1}} \frac{1}{\lambda_{i}^{\beta_{i}}}+\sum_{i \in I_{1}} \frac{\left|\nabla K\left(a_{i}\right)\right|}{\lambda_{i}}+\sum_{j \neq i \in I_{1}} \varepsilon_{i j}\right) \\
& \quad+o\left(\sum_{i=1}^{p} \frac{1}{\lambda_{i}^{\beta_{i}}}\right) .
\end{aligned}
$$


From another part, by Lemma 12 we have

$$
\begin{aligned}
& \left\langle\partial J(u), \sum_{i \in J_{1}}-2^{i} Z_{i}(u)\right\rangle \\
& \leq c \sum_{j \neq i, i \in J_{1}} 2^{i} \lambda_{i} \frac{\partial \varepsilon_{i j}}{\partial \lambda_{i}}+O\left(\sum_{i \in J_{1}} \frac{1}{\lambda_{i}^{\beta_{i}}}\right) \\
& \quad+O\left(\sum_{i \in J_{1} \cap L_{2}} \frac{\left|\left(a_{i}-y_{l_{i}}\right)_{k_{i}}\right|^{\beta_{i}-2}}{\lambda_{i}^{2}}\right) .
\end{aligned}
$$

Observe that using a direct calculation, we have

$$
\begin{aligned}
\lambda_{i} \frac{\partial \varepsilon_{i j}}{\partial \lambda_{i}} \leq-c \varepsilon_{i j}, & \text { if } \lambda_{i} \geq \lambda_{j} \text { or } \lambda_{i} \sim \lambda_{j} \\
& \text { or }\left|a_{i}-a_{j}\right| \geq \delta_{0}>0 .
\end{aligned}
$$

Since for $i<j$, we have

$$
2^{i} \lambda_{i} \frac{\partial \varepsilon_{i j}}{\partial \lambda_{i}}+2^{j} \lambda_{j} \frac{\partial \varepsilon_{i j}}{\partial \lambda_{j}} \leq-c \varepsilon_{i j}
$$

and for $i \in J_{1}$ and $j \in J_{2}$ we have $\lambda_{j} \leq \lambda_{i}$, we obtain $\lambda_{i}\left(\partial \varepsilon_{i j} / \partial \lambda_{i}\right) \leq-c \varepsilon_{i j}$. These estimates yield

$$
\begin{aligned}
& \left\langle\partial J(u), \sum_{i \in J_{1}}-2^{i} Z_{i}(u)\right\rangle \\
& \leq-c \sum_{j \neq i, i \in J_{1}, j \in J_{1} \cup J_{2}} \varepsilon_{i j}+O\left(\sum_{i \in J_{1}} \frac{1}{\lambda_{i}^{\beta_{i}}}\right) \\
& +O\left(\sum_{i \in J_{1} \cap L_{2}} \frac{\left|\left(a_{i}-y_{l_{i}}\right)_{k_{i}}\right|^{\beta_{i}-2}}{\lambda_{i}^{2}}\right) \\
& +O\left(\sum_{i \in J_{1}, j \in I_{1}} \varepsilon_{i j}\right) .
\end{aligned}
$$

It is easy to see that for any index $i \in L_{2}$, we have

$$
\frac{\left|\left(a_{i}-y_{l_{i}}\right)_{k_{i}}\right|^{\beta_{i}-2}}{\lambda_{i}^{2}} \leq \frac{\sqrt{n}}{M_{1}} \frac{\left|\left(a_{i}-y_{l_{i}}\right)_{k_{i}}\right|^{\beta_{i}-1}}{\lambda_{i}},
$$

where $k_{i}$ is defined in (45) and $M_{1}$ is large enough. Thus, we derive that

$$
\frac{\left|\left(a_{i}-y_{l_{i}}\right)_{k_{i}}\right|^{\beta_{i}-2}}{\lambda_{i}^{2}}=o\left(\frac{\left|\left(a_{i}-y_{l_{i}}\right)_{k_{i}}\right|^{\beta_{i}-1}}{\lambda_{i}}\right),
$$

for any $i \in L_{2}$.
Let $m_{1}>0$ be small enough; using Lemma 13, (63), and (55), we get

$$
\begin{aligned}
\left\langle\partial J(u), \sum_{i \in J_{1}}-2^{i} Z_{i}(u)+m_{1} \sum_{i \in J_{1} \cap L_{2}} X_{i}(u)\right\rangle \\
\leq-c\left(\sum_{j \neq i, i \in J_{1}, j \in J_{1} \cup J_{2}} \varepsilon_{i j}+\sum_{i \in J_{1}} \frac{\left|\nabla K\left(a_{i}\right)\right|}{\lambda_{i}}\right) \\
+O\left(\sum_{i \in J_{1}} \frac{1}{\lambda_{i}^{\beta_{i}}}\right)+o\left(\sum_{i=1}^{p} \frac{1}{\lambda_{i}^{\beta_{i}}}\right),
\end{aligned}
$$

and by (57) we obtain

$$
\begin{gathered}
\left\langle\partial J(u), \widetilde{W_{1}}(u)+m_{1}\left(\sum_{i \in J_{1}}-2^{i} Z_{i}(u)+m_{1} \sum_{i \in J_{1} \cap L_{2}} X_{i}(u)\right)\right\rangle \\
\leq-c\left(\sum_{i \in I_{1} \cup J_{1}} \frac{1}{\lambda_{i}^{\beta_{i}}}+\sum_{i \neq j \in I_{1}} \varepsilon_{i j}\right. \\
\left.+\sum_{j \neq i, i \in J_{1}, j \in J_{1} \cup J_{2}} \varepsilon_{i j}+\sum_{i \in I_{1} \cup J_{1}} \frac{\left|\nabla K\left(a_{i}\right)\right|}{\lambda_{i}}\right) \\
+o\left(\sum_{i=1}^{p} \frac{1}{\lambda_{i}^{\beta_{i}}}\right) .
\end{gathered}
$$

We need to add the remainder indices $i \in J_{2}$. Note that $\tilde{u}:=$ $\sum_{j \in J_{2}} \alpha_{j} \delta_{j} \in V_{2}\left(\sharp J_{2}, \varepsilon\right)$. Thus using Proposition 10, we apply the associated vector field which we will denote by $\widetilde{W_{2}}$. We then have the following estimate:

$$
\begin{aligned}
& \left\langle\partial J(u), \widetilde{W_{2}}(u)\right\rangle \\
& \leq-c\left(\sum_{j \in J_{2}} \frac{1}{\lambda_{j}^{\beta_{j}}}+\sum_{i \neq j, i, j \in J_{2}} \varepsilon_{i j}+\sum_{j \in J_{2}} \frac{\left|\nabla K\left(a_{j}\right)\right|}{\lambda_{j}}\right) \\
& \quad+O\left(\sum_{j \in J_{2}, i \in J_{1}} \varepsilon_{i j}\right)+o\left(\sum_{i=1}^{p} \frac{1}{\lambda_{i}^{\beta_{i}}}\right),
\end{aligned}
$$

since $\left|a_{i}-a_{j}\right| \geq \rho$ for $j \in J_{2}$ and $i \in I_{1}$.

Let in this case $W=\widetilde{W_{1}}+m_{1}\left(\widetilde{W_{2}}+\sum_{i \in J_{1}}-2^{i} Z_{i}+\right.$ $\left.m_{1} \sum_{i \in J_{1} \cap L_{2}} X_{i}\right)$.

From (65) and (66) we obtain

$$
\langle\partial J(u), W(u)\rangle \leq-c\left(\sum_{i=1}^{p} \frac{1}{\lambda_{i}^{\beta_{i}}}+\sum_{i=1}^{p} \frac{\left|\nabla K\left(a_{i}\right)\right|}{\lambda_{i}}+\sum_{i \neq j} \varepsilon_{i j}\right) .
$$

Case 2. $u_{1}:=\sum_{i \in I_{1}} \alpha_{i} \delta_{\left(a_{i} \lambda_{i}\right)} \in V_{1}^{1}\left(\sharp I_{1}, \varepsilon\right)$ and $u_{2}:=$ $\sum_{i \notin I_{2}} \alpha_{i} \delta_{\left(a_{i} \lambda_{i}\right)} \notin V_{2}^{1}\left(\sharp I_{2}, \varepsilon\right):=\left\{u=\sum_{j=1}^{\sharp I_{2}} \alpha_{j} \delta_{\left(a_{j}, \lambda_{j}\right)}, a_{j} \epsilon\right.$ $B\left(y_{l_{j}}, \rho\right), y_{l_{j}} \in \mathscr{K}^{+}$, for all $j=1, \ldots, \sharp I_{2}$ and $\rho\left(y_{l_{1}}, \ldots, y_{\sharp I_{2}}\right)>$ $0\}$. 
Since $u_{2} \in V_{2}\left(\sharp I_{2}, \varepsilon\right)$, by Proposition 10 , we can apply the associated vector field which we will denote by $V_{1}$. We get

$$
\begin{aligned}
& \left\langle\partial J(u), V_{1}(u)\right\rangle \\
& \leq-c\left(\sum_{i \in I_{2}} \frac{1}{\lambda_{i}^{\beta_{i}}}+\sum_{i \in I_{2}} \frac{\left|\nabla K\left(a_{i}\right)\right|}{\lambda_{i}}+\sum_{i \neq j, i, j \in I_{2}} \varepsilon_{i j}\right) \\
& \quad+O\left(\sum_{i \in I_{2}, j \in I_{1}} \varepsilon_{i j}\right) .
\end{aligned}
$$

Observe that $V_{1}(u)$ does not increase the maximum of the $\lambda_{i}$ 's, $i \in I_{2}$, since $u_{2} \notin V_{2}^{1}\left(\sharp I_{2}, \varepsilon\right)$. Fix $i_{0} \in I_{2}$ and let

$$
\widetilde{J_{1}}=\left\{i \in I_{1} \text {, s.t, } \lambda_{i}^{\beta_{i}} \geq \frac{1}{2} \lambda_{i_{0}}^{n-4}\right\}, \quad \widetilde{J_{2}}=I_{1} \backslash \widetilde{J_{1}} \text {. }
$$

Using (68) and (55), we get

$$
\begin{aligned}
& \left\langle\partial J(u), V_{1}(u)\right\rangle \\
& \leq-c\left(\sum_{i \in I_{2} \cup \widetilde{J_{1}}} \frac{1}{\lambda_{i}^{\beta_{i}}}+\sum_{i \in I_{2}} \frac{\left|\nabla K\left(a_{i}\right)\right|}{\lambda_{i}}+\sum_{i \neq j, i, j \in I_{2}} \varepsilon_{i j}\right) \\
& \quad+o\left(\sum_{i=1}^{p} \frac{1}{\lambda_{i}^{\beta_{i}}}\right) .
\end{aligned}
$$

We need to add the indices $i, i \in \widetilde{J_{2}}$. Letting $\widetilde{u}:=$ $\sum_{j \in \widetilde{J}_{2}} \alpha_{j} \delta_{\left(a_{j} \lambda_{j}\right)}$, since $\widetilde{u} \in V_{1}\left(\sharp \widetilde{J}_{2}, \varepsilon\right)$, we can apply the associated vector field given by Proposition 10. Let $V_{2}$ be this vector field. By Proposition 11 we have

$$
\begin{aligned}
& \left\langle\partial J(u), V_{2}(u)\right\rangle \\
& \leq-c\left(\sum_{j \in \widetilde{J_{2}}} \frac{1}{\lambda_{j}^{\beta_{j}}}+\sum_{j \in \widetilde{J_{2}}} \frac{\left|\nabla K\left(a_{j}\right)\right|}{\lambda_{j}}+\sum_{i \neq j, i, j \in \widetilde{J_{2}}} \varepsilon_{i j}\right) \\
& \quad+O\left(\sum_{j \in \widetilde{J_{2}}, i \notin \widetilde{J_{2}}} \varepsilon_{i j}\right) .
\end{aligned}
$$

Observe that $I_{1}=\widetilde{J_{1}} \cup \widetilde{J_{2}}$ and we are in the case where for all $i \neq j \in I_{1}$, we have $\left|a_{i}-a_{j}\right| \geq \rho$. Thus by (55), we get

$$
O\left(\sum_{j \in \widetilde{J_{2}}, i \notin \widetilde{J_{2}}} \varepsilon_{i j}\right)=o\left(\sum_{i=1}^{p} \frac{1}{\lambda_{i}^{\beta_{i}}}\right)
$$

and hence

$$
\begin{aligned}
& \left\langle\partial J(u), V_{1}(u)+V_{2}(u)\right\rangle \\
& \quad \leq-c\left(\sum_{i=1}^{p} \frac{1}{\lambda_{i}^{\beta_{i}}}+\sum_{i \in I_{2} \cup \widetilde{J_{2}}} \frac{\left|\nabla K\left(a_{i}\right)\right|}{\lambda_{i}}+\sum_{i \neq j} \varepsilon_{i j}\right) .
\end{aligned}
$$

Let in this case $W=V_{1}+V_{2}+m_{1} \sum_{i \in \widetilde{J_{1}}} X_{i}(u), m_{1}$ small enough.
Using the previous estimate and Lemma 13, we find that

$$
\langle\partial J(u), W(u)\rangle \leq-c\left(\sum_{i=1}^{p} \frac{1}{\lambda_{i}^{\beta_{i}}}+\sum_{i=1}^{p} \frac{\left|\nabla K\left(a_{i}\right)\right|}{\lambda_{i}}+\sum_{i \neq j} \varepsilon_{i j}\right) .
$$

Case 3. $u_{1} \in V_{1}^{1}\left(\sharp I_{1}, \varepsilon\right)$ and $u_{2} \in V_{2}^{1}\left(\sharp I_{2}, \varepsilon\right)$.

Let $\widetilde{V_{1}}\left(\right.$ resp., $\left.\widetilde{V_{2}}\right)$ be the pseudo-gradient in $V(p, \varepsilon)$ defined by $\widetilde{V_{1}}(u)=W_{1}\left(u_{1}\right)$ (resp., $\widetilde{V}_{2}(u)=W_{2}\left(u_{2}\right)$ ) where $W_{1}$ (resp., $W_{2}$ ) is the vector field defined by Proposition 11 (resp., Proposition 10) in $V_{1}^{1}\left(\sharp I_{1}, \varepsilon\right)$ (resp., $\left.V_{2}^{1}\left(\sharp I_{2}, \varepsilon\right)\right)$ and let in this case

$$
W=\widetilde{V_{1}}+\widetilde{V_{2}}
$$

Using Proposition 10, Proposition 11, and (55) we get

$$
\langle\partial J(u), W(u)\rangle \leq-c\left(\sum_{i=1}^{p} \frac{1}{\lambda_{i}^{\beta_{i}}}+\sum_{i=1}^{p} \frac{\left|\nabla K\left(a_{i}\right)\right|}{\lambda_{i}}+\sum_{i \neq j} \varepsilon_{i j}\right) .
$$

Notice that in the first and second cases, the maximum of the $\lambda_{i}$ 's, $1 \leq i \leq p$, is a bounded function and hence the Palais-Smale condition is satisfied along the flow lines of $W$. However in the third case all the $\lambda_{i}$ 's, $1 \leq i \leq p$, will increase and go to $+\infty$ along the flow lines generated by $W$.

Subset 2. We consider the case of $u=\sum_{i=1}^{p} \alpha_{i} \delta_{i} \in V(p, \varepsilon)$, such that there exist $a_{i}$ satisfying $a_{i} \notin \cup_{y \in \mathscr{K}} B(y, \rho)$.

In this region, the construction of the pseudo-gradient $W$ proceeds exactly as the proof of Theorem 3.2, of subset 2, of [11].

Finally, observe that our pseudogradient $W$ in $V(p, \varepsilon)$ satisfies claim (i) of Theorem 9 and it is bounded, since $\left\|\lambda_{i}\left(\partial \delta_{\left(a_{i, \lambda_{i}}\right)} / \partial \lambda_{i}\right)\right\|$ and $\left\|\left(1 / \lambda_{i}\right)\left(\partial \delta_{\left(a_{i, \lambda_{i}}\right)} / \partial a_{i}\right)\right\|$ are bounded. From the definition of $W$, the $\lambda_{i}$ 's, $1 \leq i \leq p$, decrease along the flow lines of $W$ as long as these flow lines do not enter in the neighborhood of finite number of critical points $y_{l_{i}}$, $i=1, \ldots, p$, of $\mathscr{K}$ such that $\left(y_{l_{1}}, \ldots, y_{l_{p}}\right) \in \mathscr{C}_{\infty}$.

Now, arguing as in Appendix 2 of [16], see also Appendix $B$ of [18], claim (ii) of Theorem 9 follows from (i) and Proposition 5. This completes the proof of Theorem 9.

Corollary 14. Let $p \geq 1$. The critical points at infinity of $J$ in $V(p, \varepsilon)$ correspond to

$$
\left(y_{l_{1}}, \ldots, y_{l_{p}}\right)_{\infty}:=\sum_{i=1}^{p} \frac{1}{K\left(y_{l_{i}}\right)^{(n-4) / 2}} \delta_{\left(y_{l_{i}}, \infty\right)},
$$

where $\left(y_{l_{1}}, \ldots, y_{l_{p}}\right) \in \mathscr{C}_{\infty}$. Moreover, such a critical point at infinity has an index equal to $i\left(y_{l_{1}}, \ldots, y_{l_{p}}\right)_{\infty}=p-1+\sum_{i=1}^{p} n-$ $\tilde{i}(y)$.

\section{Proof of Theorem 1}

We prove the existence result by contradiction. Assume that $J$ has no critical point in $V_{\eta}\left(\Sigma^{+}\right)$. It follows from Corollary 14 
that the critical points at infinity of the associated variational problem are in one to one correspondence with the elements of $\mathscr{C}_{\infty}$ defined in (13).

Notice that, just like for usual critical points, it is associated with each critical point at infinity $w_{\infty}$ of $J$ stable and unstable manifolds $W_{s}^{\infty}\left(w_{\infty}\right)$ and $W_{u}^{\infty}\left(w_{\infty}\right)$ (see [16, pages 356-357]). These manifolds can be easily described once a finite-dimensional reduction like the one we performed in Section 3 is established.

For any $w_{\infty}=\left(y_{i_{1}}, \ldots, y_{i_{p}}\right) \in \mathscr{C}_{\infty}$, let $c(w)_{\infty}=$ $S_{n}\left(\sum_{j=1}^{p} 1 / K\left(y_{i_{j}}\right)^{(n-4) / 2}\right)^{4 / n}$ denote the associated critical value. Here we choose to consider a simplified situation, where for any $w_{\infty} \neq w_{\infty}^{\prime}, c(w)_{\infty} \neq c\left(w^{\prime}\right)_{\infty}$, and thus order the $c(w)_{\infty}$ 's, $w_{\infty} \in \mathscr{C}_{\infty}$ as

$$
c\left(w_{1}\right)_{\infty}<\cdots<c\left(w_{k_{0}}\right)_{\infty} .
$$

By using a deformation lemma (see [22, proposition 7.24 and Theorem 8.2]), we know that if $c\left(w_{k-1}\right)_{\infty}<a<c\left(w_{k}\right)_{\infty}<$ $b<c\left(w_{k+1}\right)_{\infty}$, then

$$
J_{b} \simeq J_{a} \cup W_{u}^{\infty}\left(w_{k}\right)_{\infty},
$$

where $J_{b}=\left\{u \in V_{\eta}\left(\Sigma^{+}\right), J(u) \leq b\right\}$ and $\simeq$ denotes retracts by deformation.

We apply the Euler-Poincaré characteristic of both sides of (79); we find that

$$
\chi\left(J_{b}\right)=\chi\left(J_{a}\right)+(-1)^{i\left(w_{k}\right)_{\infty},}
$$

where $i\left(w_{k}\right)_{\infty}$ denotes the index of the critical point at infinity $\left(w_{k}\right)_{\infty}$. Let

$$
\begin{aligned}
b_{1}<c\left(w_{1}\right)_{\infty}= & \min _{u \in V_{\eta}\left(\Sigma^{+}\right)} J(u)<b_{2}<c\left(w_{2}\right)_{\infty} \\
& <\cdots<b_{k_{0}}<c\left(w_{k_{0}}\right)_{\infty}<b_{k_{0}+1} .
\end{aligned}
$$

Since we have assumed that (4) has no solution, $J_{b_{k_{0}+1}}$ is a retard by deformation of $V_{\eta}\left(\Sigma^{+}\right)$. Therefore $\chi\left(J_{b_{k_{0}+1}}\right)=1$, since $V_{\eta}\left(\Sigma^{+}\right)$is a contractible set. Now using $(80)$, we derive after recalling that $\chi\left(J_{b_{1}}\right)=\chi(\emptyset)=0$

$$
1=\sum_{j=1}^{k_{0}}(-1)^{i\left(w_{j}\right)_{\infty}} .
$$

Hence if (82) is violated, $J$ has a critical point in $V_{\eta}\left(\Sigma^{+}\right)$.

Now, arguing as in the proof of theorem of [18, pages 659660 , we prove that such a critical point is positive.

To prove the multiplicity part of the statement, we observe that it follows from Sard-Smale theorem that for generic $K$ 's, the solutions to (7) are all nondegenerate, in the sense that the associated linearized operator does not admit zero as eigenvalue. We need to introduce the following lemma extracted from [11].

Lemma 15 (see [11, Section 3.2]). Let $w$ be a solution to (7). Assume that the function $K$ satisfies condition $(f)_{\beta}$, with $(n-$ 4) $/ 2<\beta \leq n-4$; then for each $p \in \mathbb{N}^{*}$, there are neither critical points nor critical points at infinity in $V(p, \varepsilon, w)$.
Once the existence of mixed critical points at infinity is ruled out, it follows from the previous arguments that

$$
V_{\eta}\left(\Sigma^{+}\right) \simeq \bigcup_{j=1}^{k_{0}} W_{u}^{\infty}\left(w_{j}\right)_{\infty} \cup \bigcup_{w, \partial J(w)=0} W_{u}(w) .
$$

Now using the Euler-Poincaré theorem, we derive that

$$
1=\sum_{j=1}^{k_{0}}(-1)^{i\left(w_{j}\right)_{\infty}}+\sum_{w, \partial J(w)=0}(-1)^{\operatorname{morse}(w)} .
$$

Hence our theorem follows.

\section{A General Existence Result}

In this last section of this paper, we give a generalization of Theorem 1. Namely, in view of the result of Theorem 1, one may think about the situation where the degree $d$ given in Theorem 1 is equal to zero; that is, the total sum in $d$ is equal to 1 , but a partial one is not equal to 1 . A natural question arises: is it possible in this case to use such information to derive an existence result? In the following theorem we give a partial answer to this question.

Theorem 16. Let $K$ be a function satisfying $\left(A_{1}\right)$ and $(f)_{\beta}$, with $1<\beta \leq n-4$. If there exists $k \in \mathbb{N}$ such that

$$
\begin{gathered}
\text { (1) } \forall w_{\infty} \in \mathscr{C}_{\infty}, \quad i(w)_{\infty} \neq k+1 \\
\text { (2) } \sum_{w_{\infty} \in \mathscr{C}_{\infty}, i\left(w_{\infty}\right) \leq k}(-1)^{i\left(w_{\infty}\right)} \neq 1,
\end{gathered}
$$

then the problem (7) has at least one solution.

Moreover for generic $K$ 's, if $(n-4) / 2<\beta \leq n-4$, then the number of solutions is lower bounded by

$$
\left|1-\sum_{w_{\infty} \in \mathscr{C}_{\infty}, i\left(w_{\infty}\right) \leq k}(-1)^{i\left(w_{\infty}\right)}\right| .
$$

Let $l_{\sharp}$ be the maximal index over all elements of $\mathscr{C}_{\infty}$. Please observe that the integer $k=l_{\sharp}$ satisfies (1) of Theorem 16; it follows that Theorem 1 is a corollary of Theorem 16.

Proof of Theorem 16. We set

$$
X_{k}^{\infty}=\bigcup_{x_{\infty} \in \mathscr{C}_{\infty}, i(x)_{\infty} \leq k} \overline{W_{u}^{\infty}\left(x_{\infty}\right)},
$$

where $\overline{W_{u}^{\infty}\left(x_{\infty}\right)}$ is the closure of the unstable manifolds of $x_{\infty}$. Observe that $X_{k}^{\infty}$ is a stratified set of top dimension $k$, which is contractible set in $V_{\eta}\left(\Sigma^{+}\right)$, since $V_{\eta}\left(\Sigma^{+}\right)$is a contractible set. Let $U$ denote the image of such a contraction. To prove the first part of Theorem 16, arguing by contradiction, we assume that (7) has no solution. Using the pseudo-gradient constructed in Theorem 9, we can deform $U$. By transversality arguments, we can assume that such a deformation avoids all critical points at infinity of index 
greater or equal to $k+1$. Note that, from assumption (1) of Theorem 16, there is no critical point at infinity with index $k+1$. It follows then from a theorem of Bahri and Rabinowitz [22] that

$$
U \simeq X_{k}^{\infty}
$$

Hence from the Euler-Poincaré theorem, we get

$$
1=\chi\left(X_{k}^{\infty}\right)=\sum_{w_{\infty} \in C_{\infty}, i\left(w_{\infty}\right) \leq k}(-1)^{i\left(w_{\infty}\right)},
$$

which is a contradiction with assumption (2) of Theorem 16.

Regarding the multiplicity result, we observe that for generic $K$ 's the functional $J$ admits only nondegenerate critical points. Hence by Lemma 15, the set $U$ will be deformed into

$$
U \simeq X_{k}^{\infty} \cup \bigcup_{w \in C_{k}} W_{u}(w),
$$

where $C_{k}$ denotes the set of the critical points of $J$ of Morse index less than or equal to $k$, which are dominated by $U$. Finally by using the Euler-Poincaré theorem, the proof of Theorem 16 follows.

\section{References}

[1] S. M. Paneitz, "A quartic conformally covariant differential operator for arbitrary pseudo-Riemannian manifolds," SIGMA. Symmetry, Integrability and Geometry. Methods and Applications, vol. 4, article 036, 2008.

[2] T. P. Branson, "Differential operators canonically associated to a conformal structure," Mathematica Scandinavica, vol. 57, no. 2, pp. 293-345, 1985.

[3] Z. Djadli, E. Hebey, and M. Ledoux, "Paneitz-type operators and applications," Duke Mathematical Journal, vol. 104, no. 1, pp. 129-169, 2000.

[4] Sun-Y.A. Chang and P. C. Yang, "Extremal metrics of zeta function determinants on $S^{n}$-manifolds," Annals of Mathematics, vol. 142, no. 1, pp. 171-212, 1995.

[5] Z. Djadli and A. Malchiodi, "Existence of conformal metrics with constant Q-curvature," Annals of Mathematics, vol. 168, no. 3, pp. 813-858, 2008.

[6] M. J. Gursky, "The principal eigenvalue of a conformally invariant differential operator, with an application to semilinear elliptic PDE," Communications in Mathematical Physics, vol. 207, no. 1, pp. 131-143, 1999.

[7] J. Wei and X. Xu, "On conformal deformations of metrics on $S^{n}$," Journal of Functional Analysis, vol. 157, no. 1, pp. 292-325, 1998.

[8] W. Abdelhedi and H. Chtioui, "On the prescribed Paneitz curvature problem on the standard spheres," Advanced Nonlinear Studies, vol. 6, no. 4, pp. 511-528, 2006.

[9] A. Bensouf and H. Chtioui, "Conformal metrics with prescribed Q-curvature on $S^{n}$," Calculus of Variations and Partial Differential Equations, vol. 41, no. 3-4, pp. 455-481, 2011.

[10] H. Chtioui and A. Rigane, "On the prescribed Q-curvature problem on $S^{n}$," Comptes Rendus Mathématique. Académie des Sciences. Paris, vol. 348, no. 11-12, pp. 635-638, 2010.

[11] H. Chtioui and A. Rigane, "On the prescribed Q-curvature problem on $S^{n}$," Journal of Functional Analysis, vol. 261, no. 10, pp. 2999-3043, 2011.
[12] Z. Djadli, A. Malchiodi, and M. O. Ahmedou, "Prescribing a fourth order conformal invariant on the standard sphere. I. A perturbation result," Communications in Contemporary Mathematics, vol. 4, no. 3, pp. 375-408, 2002.

[13] Z. Djadli, A. Malchiodi, and M. O. Ahmedou, "Prescribing a fourth order conformal invariant on the standard sphere. II. Blow up analysis and applications," Annali della Scuola Normale Superiore di Pisa, vol. 1, no. 2, pp. 387-434, 2002.

[14] V. Felli, "Existence of conformal metrics on $S^{n}$ with prescribed fourth-order invariant," Advances in Differential Equations, vol. 7, no. 1, pp. 47-76, 2002.

[15] A. Bahri, Critical Points at Infinity in Some Variational Problems, vol. 182 of Pitman Research Notes in Mathematics Series, Longman Scientific \& Technical, Harlow, UK, 1989.

[16] A. Bahri, "An invariant for Yamabe-type flows with applications to scalar-curvature problems in high dimension," Duke Mathematical Journal, vol. 81, no. 2, pp. 323-466, 1996.

[17] C.-S. Lin, "A classification of solutions of a conformally invariant fourth order equation in $\mathbb{R}^{n}$," Commentarii Mathematici Helvetici, vol. 73, no. 2, pp. 206-231, 1998.

[18] M. Ben Ayed, Y. Chen, H. Chtioui, and M. Hammami, "On the prescribed scalar curvature problem on $S^{n}$-manifolds," Duke Mathematical Journal, vol. 84, no. 3, pp. 633-677, 1996.

[19] E. Hebey and F. Robert, "Asymptotic analysis for fourth order Paneitz equations with critical growth," Advances in Calculus of Variations, vol. 4, no. 3, pp. 229-275, 2011.

[20] M. Ben Ayed and K. El Mehdi, "The Paneitz curvature problem on lower-dimensional spheres," Annals of Global Analysis and Geometry, vol. 31, no. 1, pp. 1-36, 2007.

[21] R. Ben Mahmoud and H. Chtioui, "Prescribing the scalar curvature problem on higher-dimensional manifolds," Discrete and Continuous Dynamical Systems A, vol. 32, no. 5, pp. 18571879, 2012.

[22] A. Bahri and P. H. Rabinowitz, "Periodic solutions of Hamiltonian systems of three body type," Annales de l'Institut Henri Poincaré. Analyse Non Linéaire, vol. 8, no. 6, pp. 561-649, 1991. 


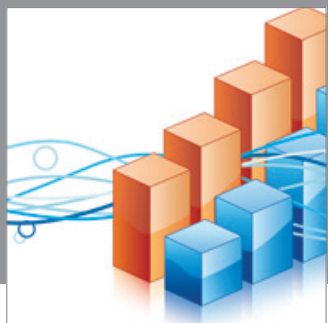

Advances in

Operations Research

mansans

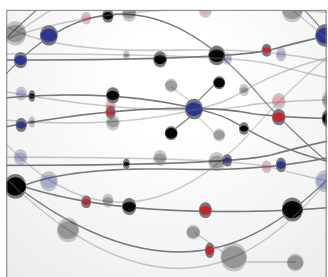

The Scientific World Journal
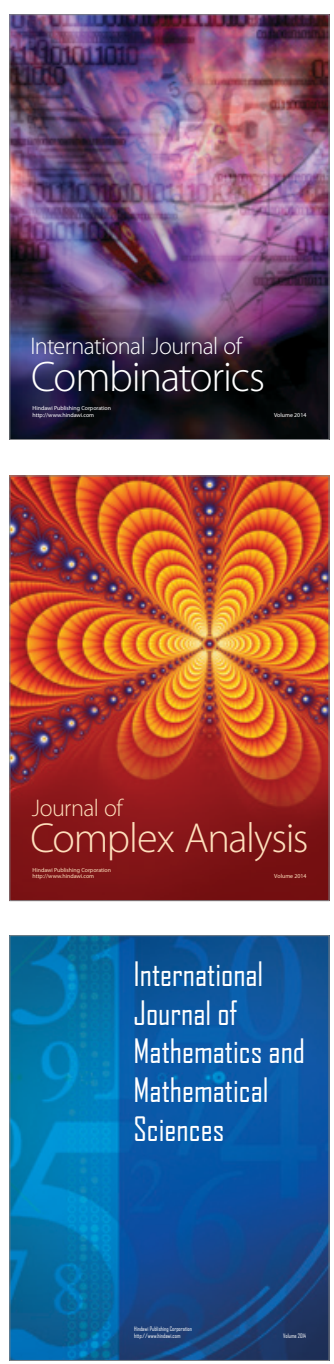
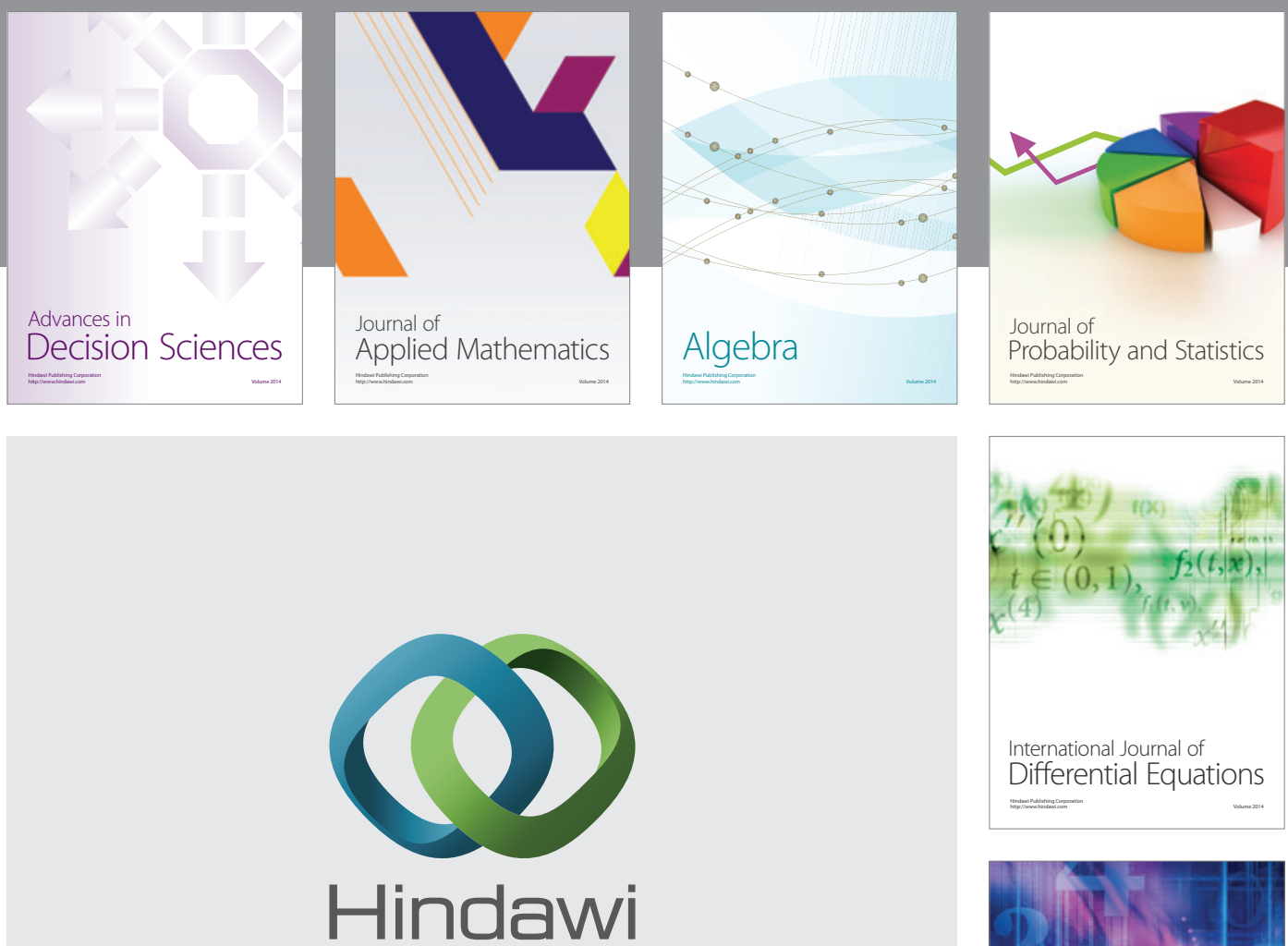

Submit your manuscripts at http://www.hindawi.com
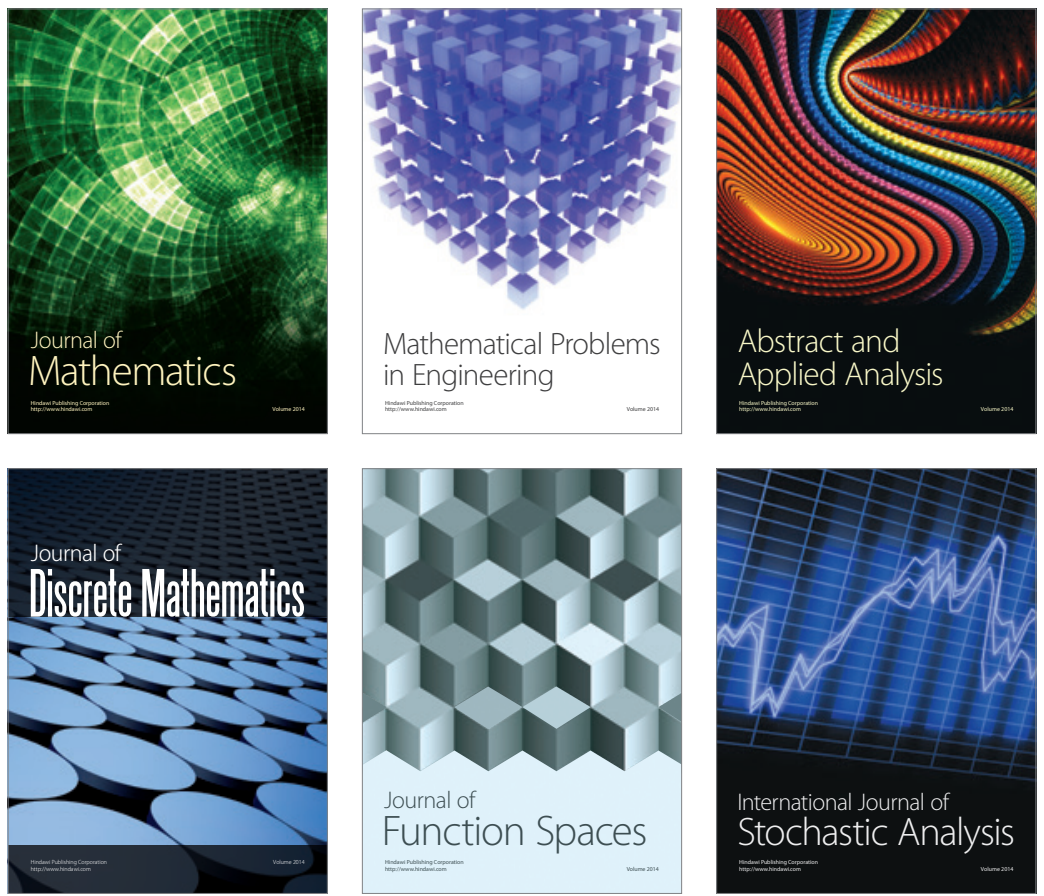

Journal of

Function Spaces

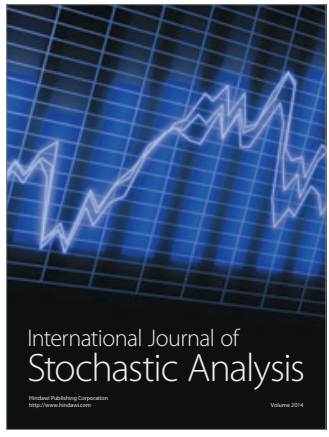

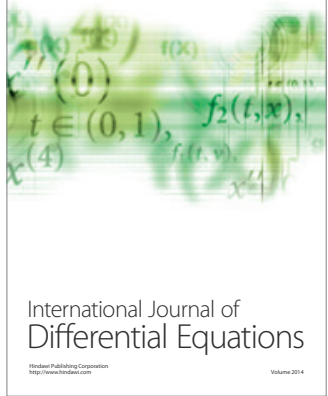
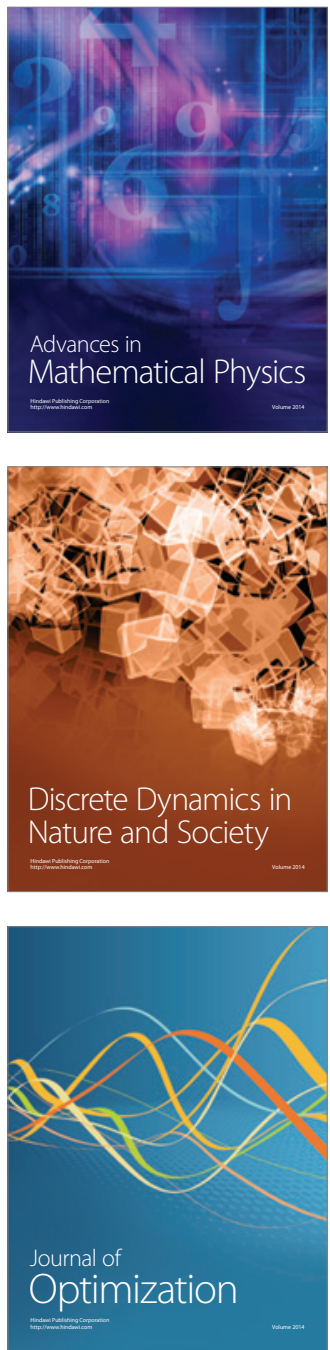